REVISTA DE CIENCIAS FORENSES DE HONDURAS
Revista de Ciencias Forenses de Honduras ISSN: 2412-8058

ISSN: 2413-1067

revistacienciasforenseshnd@gmail.com

Dirección de Medicina Forense de Honduras Honduras

Mejía Dueñas, Carlos Abraham; Matamoros, Mireya; Díaz, Roxana; Villanueva, Semma Julissa Utilización de signos inespecíficos en el diagnóstico de asfixia por sumersión. Caso y Revisión Bibliográfica.

Revista de Ciencias Forenses de Honduras, vol. 4, núm. 1, 2018, Enero-Junio, pp. 17-27

Dirección de Medicina Forense de Honduras

Honduras 


\section{Utilización de signos inespecíficos en el diagnóstico de asfixia por sumersión. Caso y Revisión Bibliográfica.}

\section{Use of nonspecific signs in the diagnosis of asphyxia by submersion. Case and Bibliographic review}

Carlos Abraham Mejía Dueñas ${ }^{1}$, Mireya Matamoros², Roxana Díaz ${ }^{3}$, Semma Julissa Villanueva ${ }^{4}$.

\footnotetext{
1,3 Médico Residente Postgrado de Medicina Legal y Forense, ${ }^{2}$ Investigación en Ciencias Forenses ${ }^{4}$ Posgrado en Medicina Legal y Forense UNAH y Dirección de Medicina Forense
}

Correspondencia a: carlosmejiaduenas@gmail.com

Recibido: noviembre 2017, Aceptado: septiembre 2018.

\section{CITAR COMO}

Mejía-dueñas C, Matamoros M, Díaz R, Villanueva SJ. Utilización de signos inespecíficos en el diagnóstico de asfixia por sumersión. Caso y Revisión Bibliográfica. Rev. cienc. forenses Honduras. 2018; 4(1): 17-27.

Los autores declaran que no tienen conflicto de intereses en la realización de este trabajo.

\section{RESUMEN}

Las asfixias por sumersión representan un enorme reto para los médicos no solo por la dificultad para llegar a un diagnóstico definitivo de la causa de muerte, sino por la dificultad para determinar las circunstancias en las que se han producido; cuando el cadáver además está en putrefacción, representan un verdadero desafío; por lo que en algunos de estos casos, los forenses se auxilian de hallazgos inespecíficos como el fenómeno del diente rosado y la hemorragia de la lámina cribosa del etmoides para establecer la causa de muerte. Presentamos el caso de un menor de edad que se encontró en las riveras de un río, con antropofagia cadavérica extensa y en estado de putrefacción, la causa de muerte se concluyó en base a los hallazgos de diente rosado y hemorragia de la lámina cribosa del etmoides, como asfixia por sumersión. Se analizó la validez de utilizar ambos signos como indicadores asfícticos.

\section{PALABRAS CLAVE}

Asfixia por sumersión, Diente rosado, Signo de Vargas Alvarado, Reporte de caso, Revisión bibliográfica.
ABSTRACT
In the forensic field asphyxiation by drowning represent a great challange for the medical examiners not only for the difficulty to determine the cause of death but for the difficulty to define the circumstances in which the death has occur; and even worse when the body is in advanced stage of decomposition, the challenge is even bigger; therefore in some cases the medical examiners use unespecific findings such as the pink teeth and the ethmoidal haemorrhage to determine the cause of death. We present case report of a child's body found near by the river, 
with extended anthropophagy and advanced decomposition stage, the cause of death was concluded based on the pink teeth findings and the ethmoidal haemorrhage and it was defined as asphyxiation by drowning. We analized the validation to use both signs as asphyxial indicators

\section{KEYWORD}

Drowning, asphyxia, pink teeth, Vargas Alvarado's sign, case report, literature review.

\section{PRESENTACIÓN DEL CASO}

Línea de tiempo

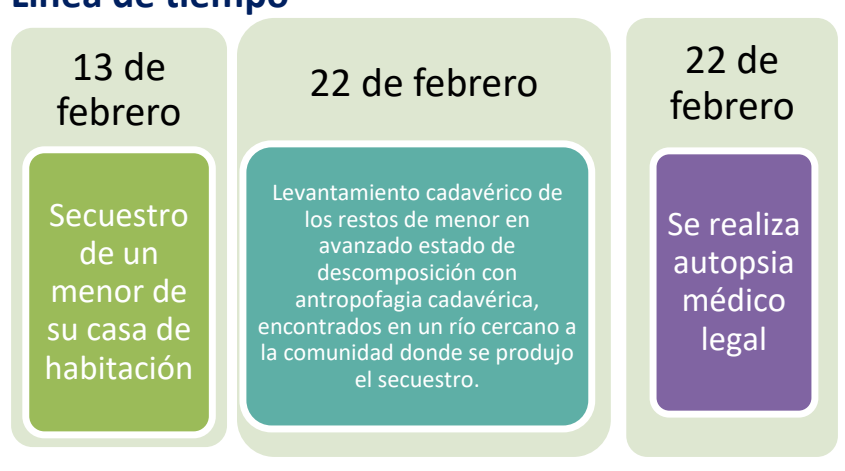

\section{Información de la Víctima}

Datos de investigación revelan a un menor de seis años que vivía con su madre, dos hermanos y su padrastro, en un ambiente familiar muy inestable, por lo que la pareja se separó, tiempo después de la separación, el padrastro, regresó a la casa de habitación para sustraer a dos de los menores, uno de los cuales era su hija biológica y el otro su hijastro. Una semana después regresó con la niña, refiriendo desconocer donde se encontraba su hijastro. Las autoridades realizaron una búsqueda exhaustiva, encontrando el cadáver de un menor, en un espacio abierto, húmedo, de clima cálido en las riveras de un rio. Los restos presentaban antropofagia y avanzado estado de descomposición.

\section{Hallazgos de autopsia}

Cadáver en avanzado estado de descomposición (fase enfisematosa), con áreas de antropofagia extensas, ausencia de órganos y tejidos en el cráneo, tórax y abdomen, con intervalo postmortem entre 8 y 10 días. En base a los estudios antropológicos y odontológicos se estableció que correspondían a un menor de seis años. El estudio radiológico descartó fracturas. Se encontró hemorragia de la lámina cribosa del etmoides. La autopsia oral reveló la presencia del fenómeno de diente rosado en la corona del segundo molar inferior derecho. Estudios de ADN confirmaron que se trataba del menor secuestrado.

La causa de muerte se concluyó como "asfixia por sumersión" sustentado en los hallazgos de autopsia como la presencia de diente rosado y hemorragia de la lámina cribosa del etmoides o signo de Vargas Alvarado, la manera se concluyó como homicida.

\section{INTRODUCCIÓN}

En el ámbito forense las asfixias por sumersión representan un enorme reto para los médicos no solo por la dificultad para llegar a un diagnóstico definitivo de la causa de la muerte, sino por la dificultad para determinar las circunstancias en las que se han producido; si el cadáver además está en fase de putrefacción, representan un verdadero desafío ${ }^{1,2}$, por lo que el forense debe auxiliarse de toda la información disponible y la evaluación de información proveniente de fuentes extra autopsia puede ser invaluable para la conclusión sobre la causa y manera de muerte en estos casos. Presentamos a un menor encontrado en avanzado estado de descomposición (fase enfisematosa), en las riveras de un rio cercano a su domicilio, con áreas de antropofagia extensas, ausencia de órganos y tejidos en el cráneo, tórax y abdomen, estado de descomposición (fase enfisematosa), en las riveras de un rio cercano a su domicilio, con áreas de antropofagia extensas, ausencia de órganos y tejidos en el cráneo, tórax y abdomen, los 
estudios radiológicos descartaron trauma óseo, la autopsia reveló hemorragia de la lámina cribosa del etmoides o signo de Vargas Alvarado; y presencia del fenómeno de diente rosado, en la corona del segundo molar inferior derecho; en la autopsia oral. La causa de muerte se concluyó como asfixia por sumersión, sustentada en estos hallazgos. Ante esta conclusión nos planteamos la interrogante, sobre ¿Cuál es la validez de utilizar hallazgos inespecíficos como la hemorragia de la lámina cribosa del etmoides ${ }^{3} y$ el diente rosado ${ }^{4}$, como indicadores de asfixia por sumersión? Se realizó una revisión bibliográfica a fin de analizar los fundamentos y vigencia de ambos signos y su asociación con la asfixia por sumersión.

\section{Metodología de búsqueda}

Se realizó una búsqueda en pubmed, medline, google académico, libros de texto, así como la revisión de los artículos citados, usando las palabras clave: asfixia por sumersión, mecanismos de asfixia, hemorragia de la lámina cribosa del etmoides, signo de Vargas Alvarado, diente rosado.

\section{CONCEPTUALIZACIÓN}

\section{Asfixia}

El termino asfixia se utilizó inicialmente para designar la falta de pulso o para designar las muertes repentinas acompañadas de paro cardiaco. Posteriormente se ha relacionado con la dificultad o detención de la función respiratoria y con tal significado persiste en la literatura médico-legal. Savaugeu lo define como la situación en la cual el cuerpo no recibe o utiliza las cantidades adecuadas de oxígeno ${ }^{5-8}$.

En el contexto médico legal la clasificación de asfixias y la definición de los subtipos varía de acuerdo al autor o libro de texto consultado, Savaugeu en el 2010 propuso la clasificación en cuatro grupos $^{8}$ :
1.-Asfixia por sofocación, que a su vez se subdivide compresión toracoabdominal, carencia de aire respirable; subdividiéndose en confinamiento y aplastamiento.

2.-Asfixias por estrangulación que incluye: estrangulación por ligaduras, ahorcamiento y estrangulación manual.

3.-Asfixias mecánicas que incluye la posicional y la traumática.

4.-Asfixia por sumersión.

En el 2012 Joon y colaboradores propusieron una nueva clasificación que incluye las tres primeras categorías propuestas por Savaugeu, pero que sustituye la cuarta categoría de ahogamiento por asfixias complicadas ${ }^{9}$.

\section{Asfixia por sumersión}

Se han descrito múltiples definiciones de asfixia por sumersión, las cuales pueden ser confusas o tener otro significado en el contexto médico legal. En el 2002 se celebró en Amsterdam el primer Congreso Mundial sobre asfixia por sumersión, a partir del cual se elaboró una guía de recomendaciones y definiciones de sumersión, que se conocen como el "estilo Utstein" y que fue revisado en el $2015^{10,11}$, aquí se definió la sumersión como "el proceso de experimentar insuficiencia respiratoria por la sumersión o inmersión en un medio líquido, de modo que el fluido que rodea a la víctima llega a bloquear las vías respiratorias e inhibir la respiración. La víctima puede vivir o morir después de este proceso"12.

Según Concheiro y Suárez ${ }^{6}$, la sumersión, en sentido médico-legal, es la muerte o el trastorno patológico producido por la introducción de un medio líquido, habitualmente agua, en las vías respiratorias, estos autores recomiendan no incluir bajo el concepto de sumersión los producidos por la penetración de material semilíquido o pastoso en las vías aéreas, ya que los trastornos fisiopatológicos resultantes coinciden más con los que se producen en los 
casos de sofocación por obstrucción intrínseca de las vías respiratorias.

\section{MECANISMOS FISIOPATOLÓGICOS DE LAS ASFIXIAS POR SUMERSION}

Calabuig describe que la anoxia anoxica es el mecanismo clásico por el que se explica la muerte por sumersión y que a partir de datos experimentales además se ha concedido importancia a los cambios electrolíticos; describe que cuando la sumersión se produce en agua dulce produce hipervolemia, hemolisis y una agresión anoxica en el miocardio, con posterior fibrilación ventricular, sin embargo cuando la sumersión se produce en agua salada hay hemoconcentración y edema pulmonar, con daño cardiaco subsecuente ${ }^{6}$.

Schilling y Bartollini ${ }^{13}$ proponen que actualmente la patofisiología de la sumersión es definida de manera diferente, ellos proponen que cuando la vía aérea de la víctima está bajo la superficie del líquido, inicialmente la víctima trata de contener la respiración, seguidamente los esfuerzos respiratorios involuntarios evolucionan, comenzando con las contracciones diafragmáticas, alternativamente, el líquido ingresa a la faringe a través de la nariz. En cualquier caso, hay menor aspiración, causando muy a menudo laringoespasmo reflejo-mediado, por tanto, la respiración no es posible durante este período, y se desarrolla hipoxia, hipercapnia y acidosis. Cuando cesa el laringoespasmo, la respiración no puede mantenerse voluntariamente y se produce una aspiración mayor. A menudo, grandes cantidades de líquido ingresan al estómago durante este período. Como no se puede inspirar más oxígeno, la hipoxia se desarrolla rápidamente. La pO2 arterial disminuye en aproximadamente $6 \mathrm{mmHg}$ por minuto; a continuación, se desarrolla la inconsciencia, finalmente, la hipoxia cerebral produce la muerte; el esquema que muestra la Figura 1, resume el mecanismo propuesto por estos autores. Se han descrito mecanismos fisiopatológicos diferenciados a partir de modelos animales, respecto a si el ahogamiento se realiza en agua dulce o salada, sin embargo, algunos autores proponen que esta diferenciación carece de importancia en los humanos, pero si podría tener significancia desde la perspectiva médico legal ${ }^{14,15}$.

Bierens y Lunetta señalan que en general la fisiología del ahogamiento se relaciona con dos eventos diferentes: inmersión y la sumersión. La inmersión involucra respuestas cardiorrespiratorias integradas a la piel y temperatura corporal profunda, incluyendo shock frío, incapacidad física e hipovolemia, como precursores de colapso y sumersión. La fisiología de la sumersión incluye miedo a ahogarse, respuesta de buceo, conflicto autonómico superior, reflejos de las vías respiratorias, aspiración de agua y deglución, emesis y trastornos electrolíticos. El resultado de la sumersión está determinado por lesión cardiaca, pulmonar y neurológica ${ }^{16}$.

\section{Abordaje médico legal}

Determinar la causa y manera de muerte en una asfixia por sumersión conlleva importantes dificultades; por lo que el abordaje de los casos debe realizarse de manera integral analizando pormenorizadamente la escena, la presencia de testigos, los antecedentes patológicos de la víctima, problemas para encontrar o recuperar el cadáver, el estado del mismo al momento del hallazgo (putrefacto o no), alteraciones producidas por actividad animal etc. 
Figura 1: Fisiopatología de la sumersión según Bartollini y Schilling

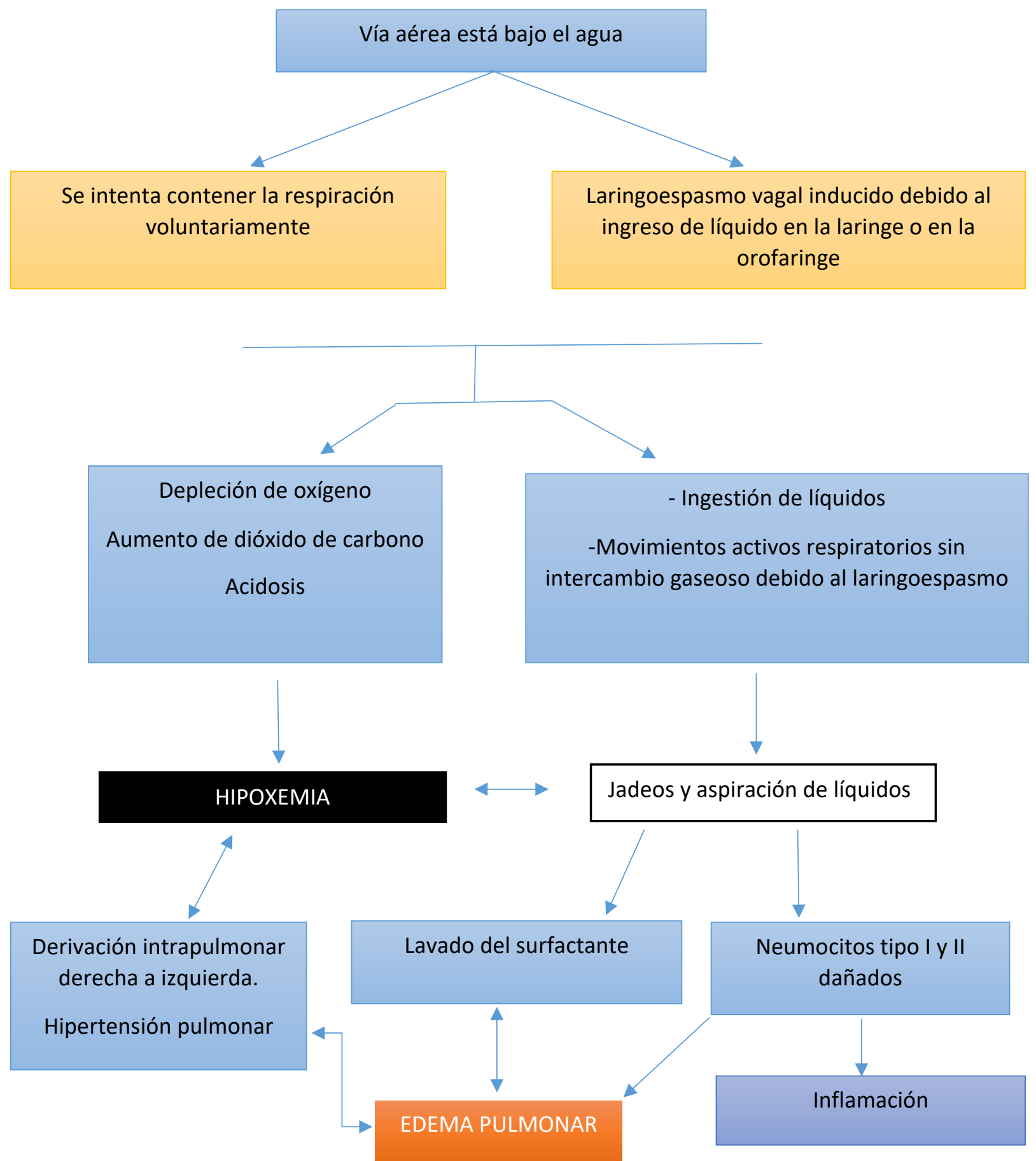


La etiología de la muerte en un cadáver recuperado del agua podría ser ahogamiento accidental, homicida o suicida; o ahogamiento con precipitación debido a una causa natural por enfermedad, por ejemplo estudios indican que los epilépticos tienen entre 15 y 19 veces más riesgo de morir ahogados que las personas que no padecen epilepsia ${ }^{17}$, alternativamente el ahogamiento podría ser secundario a una enfermedad de base como ciertas canalopatías como la LQT1 que se ha descrito causa ciertas arritmias mientras se nada ${ }^{17,18}$.

Ante un cadáver recuperado del agua el forense debe contestar al menos las siguientes preguntas 18:

-¿Quién es la víctima?

-¿Estaba viva antes de entrar en el agua?

- ¿Falleció por una causa natural o violenta antes del ingreso en el agua? -¿Le sobrevino una muerte natural o violenta en el agua? -¿Sufrió una muerte por inhibición en el agua? -¿Sufrió un cuadro de hipotermia en el agua? -¿Precipitó la hipotermia el fallecimiento en el agua?

-¿Por qué motivo fue incapaz de sobrevivir en el agua?

-¿Pudo influir en el desenlace algún tóxico? -¿Cuánto tiempo ha permanecido en el agua?

Con el fin de contestar estas y otras interrogantes el forense debe recurrir a una serie de estudios cuya aplicación depende del estado en el que se encontró el cadáver.

\section{HALLAZGOS DE AUTOPSIA}

\section{1.-Examen externo}

En el examen externo del cadáver recuperado del agua, independientemente de su mecanismo de muerte se pueden encontrar una serie de alteraciones ${ }^{6,18-20}$.

\section{Signos propios de la reacción vital:}

-Hongo de espuma que constituye un elemento indicativo, pero no especifico.

-Espasmo cadavérico con dedos, fuertemente flexionados, con restos vegetales, arena, alga o cualquier elemento macro compatible con el lugar de hallazgo o de donde se produjo la sumersión.

-Cutis anserina, enfriamiento corporal precoz, maceración cutánea.

-Evolución de la putrefacción cadavérica; influenciada por la temperatura del medio en que el cadáver está sumergido, mientras que el cadáver permanece sumergido, la putrefacción parece seguir un ritmo más lento que si se encontrase al aire libre, una vez recuperado el cadáver del agua, la putrefacción se acelera considerablemente.

-Presencia de lesiones externas: Pueden dar lugar a problemas de interpretación. En algunos casos, las lesiones tienen un marcado carácter vital (lesiones de etiología homicida); en otros casos, las lesiones obedecen a un mecanismo contusivo grave al caer sobre un fondo rocoso o sólido, aunque pueden encontrarse lesiones postmortem producto del arrastre del cadáver por la corriente de agua.

\section{2.-Examen interno}

Los hallazgos de autopsia dependen del mecanismo de la muerte ${ }^{20}$ :

Cuando el mecanismo de la muerte es el denominado sumersión-inhibición, en el que el individuo queda en muerte aparente dentro del agua debido al reflejo inhibitorio vagal que produce una parada brusca de las funciones cardio-respiratorias, los hallazgos que podemos 
encontrar son escasos, salvo una congestión inespecífica generalizada.

Cuando el mecanismo de la muerte es el denominado sumersión-asfixia simple, sin paso de agua a los pulmones por probable laringoespasmo, hallaremos agua en el aparato digestivo y signos inespecíficos de los cuadros de asfixia (Síndrome asfíctico).

Cuando el mecanismo de la muerte es el denominado sumersión-ahogamiento propiamente dicho, con penetración de agua en las vías respiratorias. Pueden observarse pulmones congestivos, con aumento de volumen, equimosis o manchas de Paltauf, de localización subpleural, las cuales están relacionadas con el proceso asfíctico y al componente traumático ejercido por la penetración del agua.

\section{Hallazgos histopatológicos}

Shkrum y Ramsay en 2007 sistematizaron los hallazgos microscópicos en los pulmones como enfisema acuoso, hemorragia alveolar, ruptura de paredes alveolares, etc., sin embargo, estos no son específicos, además, la putrefacción los desvanece rápidamente ${ }^{20}$. Otro hallazgo descrito es el peso de los pulmones y su relación con el peso del corazón y del cuerpo, sin embargo, esta relación debe usarse únicamente combinada con otros criterios diagnósticos ${ }^{21}$. Asimismo, se han descrito múltiples alteraciones en el aparato digestivo como presencia del medio de sumersión y cuerpos extraños, además de la presencia de roturas en la mucosa del fondo del estómago y se propone que la presencia de lesiones en la mucosa gástrica tiene un significado vital ${ }^{22}$.

En el corazón se describe que la tinción de las cámaras del ventrículo izquierdo, como signo diferencial en el ahogamiento en agua dulce 23, también se describe la tinción hemoglobínica de la porción proximal de la aorta como un posible marcador de la sumersión en agua dulce ${ }^{24}$.

\section{4.-Analisis de humor vítreo, LCR y presencia de diatomeas}

Los cambios bioquímicos en diferentes fluidos corporales han sido analizados en los casos de asfixias por sumersión, sin obtener resultados precisos en el diagnóstico de ahogamiento, asimismo la prueba de diatomeas ha sido ampliamente utilizada como indicador para diagnóstico de sumersión posmortem, sin embargo, se han encontrado problemas de contaminación cruzada y otras limitantes, pero aún se considera como evidencia auxiliar importante en estos $\operatorname{casos}^{19,25,26}$.

\section{Asfixia por sumersión en cuerpos putrefactos} Una limitante importante en el abordaje de las asfixias por sumersión es que la mayoría de los cuerpos son rescatados cuando ya ha aparecido la putrefacción, esto obliga a que el forense deba registrar todos los elementos potencialmente relevantes, aunque sean inespecíficos, entre los cuales encontramos:

\section{1.-La Presencia de agua en los senos} paranasales, un estudio realizado por Zivković et al. (2013) indica que el líquido libre encontrado en los senos esfenoidales (signo Svechnikov) puede considerarse como una reacción vital en los casos de sumersión sin putrefacción. En los cuerpos putrefactos recuperados del agua, una cantidad de 0,55 $\mathrm{ml}$ de líquido libre en los senos esfenoidales puede sugerir que la víctima estaba viva antes del contacto con el agua, aunque no demuestra que la sumersión sea la causa de muerte $^{27}$. 


\section{2.-Presencia de Hemorragia timpanomastoidea} y edema severo de la submucosa o signo de Niles $^{28}$. Se deduce que el esfuerzo respiratorio contra el cierre de la glotis no es el único responsable de la hemorragia timpanomastoidea que tiene lugar en los casos de muerte por sumersión. Se propone que es necesaria una membrana timpánica intacta para crear suficiente presión negativa en la cavidad del oído medio para causar una ruptura de los vasos sanguíneos y hemorragia. Por lo tanto, una hemorragia timpanomastoidea en ausencia de otitis media, traumatismo craneal, accidente cerebrovascular o diátesis hemorrágica parece ser una fuerte evidencia de apoyo a la muerte por sumersión ${ }^{19,} 29$.

3.-Fenomeno del Diente rosado: se produce debido al cambio físico que ocurre por la degradación de la hemoglobina que se filtra a la dentina, también se explica por el incremento de la presión intracapilar causada por la congestión de la sangre en zonas donde no hay pulpa dentaria; se ha observado en casos donde la muerte se produce por deficiencia de oxígeno ${ }^{30}$. Fue descrito por primera vez en el año 1829 por Thomas Bell ${ }^{31}$; actualmente se indica que su aparición postmortem depende de varios factores entre los que el ambiente húmedo; la temperatura ambiental elevada; la inmersión en agua y/o la exposición a toxinas hace que se acelere, por lo que se ha establecido que es orientativo, pero no específico de la causa de muerte y no debe tomarse como un signo patognomónico de asfixia ${ }^{32,33}$. Aunque no hay una ubicación específica de presentación se ha observado preferentemente en los dientes incisivos, caninos, y premolares y preferentemente en los cadáveres de personas jóvenes en comparación a los adultos porque la cavidad pulpar de las personas jóvenes está más vascularizada lo que permite el filtrado de la sangre y su posterior degradación ${ }^{32}$. Ciertas posiciones del cadáver favorecen su aparición como por ejemplo en decúbito ventral ${ }^{33}$. El tiempo de aparición oscila entre una y dos semanas después de la muerte. ${ }^{34}$

4.-Signo de Vargas Alvarado: Uno de los signos de asfixia por ahogamiento citados, especialmente en la Escuela Forense Costarricense es el signo de Vargas Alvarado (1972) que consiste en infiltración hemorrágica a través de las celdillas de las láminas cribosas del hueso etmoides observándose una coloración azul a ambos lados de la Crista galli; sin embargo este signo tiene la limitante que no hay estudios de validación que lo respalden y la única referencia al respecto es la descrita por Vargas Alvarado et al, posteriormente ha sido citado por otros autores, todos referidos a la misma Escuela Costarricense ${ }^{4,35}$.

\section{DISCUSIÓN}

El diagnóstico de las asfixias por sumersión, continua siendo un diagnostico difícil, especialmente en cuerpos putrefactos, este debe basarse en el análisis de información proveniente de varias fuentes: investigaciones policiales, hallazgos de autopsia, análisis microscópicos, pruebas bioquímicas, pero no únicamente sobre los hallazgos de autopsia, por lo inespecífico de los mismos; en un estudio realizado en el 2002 por Lunetta y colaboradores, después de estudiar 1590 casos de cadáveres recuperados del agua, concluyeron que sólo el $11 \%$ de todos las casos mostraban una combinación de hallazgos patológicos exclusivos de los casos de sumersión (hongo de espuma y solapamiento de los bordes anteriores de los pulmones), en $60,6 \%$ de los 
casos no se encontraron hallazgos macro morfológicos que permitieran un diagnóstico definitivo ${ }^{36}$.

Respecto al diente rosado su aparición postmortem depende de varios factores entre los que el ambiente húmedo; la temperatura ambiental elevada; la inmersión en agua y/o la exposición a toxinas hace que se acelere la degradación de la hemoglobina, por lo que no debe tomarse como un signo patognomónico de asfixia. Algunas de las condiciones predisponentes para la aparición de este fenómeno estaban presentes en el caso que presentamos por lo que no se puede establecer una relación causa-efecto, respecto a la presencia del fenómeno y la asfixia por sumersión. Referente al signo de Vargas Alvarado, debido a que no hay estudios que por ahora validen este hallazgo y los únicos estudios encontrados refieren únicamente a la Escuela Forense Costarricense, es recomendable no darle tanto peso.

Una autopsia completa, con exámenes histopatológicos y toxicológicos completos son importantes para determinar si la muerte realmente siguió a la inmersión en el agua o para ver si alguna enfermedad natural o uso de sustancias ha contribuido o causado la muerte. A la luz de lo expuesto es recomendable abandonar la práctica de conferir a signos inespecíficos la suficiente certeza para determinar la causa de muerte, por lo que en ausencia de más información los casos deben concluirse como indeterminada desde el punto de vista médicolegal. Se espera que la información expuesta contribuya a ilustrar al especialista en medicina forense sobre los conceptos más actuales de las asfixias por sumersión.

\section{REFERENCIAS BIBLIOGRAFICAS}

1.-Papadodima SA, Athanaselis SA, Skliros E, Spiliopoulou CA. Forensic investigation of submersion deaths. Int J Clin Pract. 2010;64(1):75-83. doi: 10.1111 / j.17421241.2008.01890. x. Epub 2009, 16 de febrero.

2.- Piette $M H$, De Letter EA. Drowning: still a difficult autopsy diagnosis. Forensic Sci Int. 2006 Nov 10;163(1-2):1-9. Epub 2005 Dec 27.

3.-Manoilescu I. Et al. Post-mortem changes in teeth-forensic issues. International Journal of Medical Dentistry. 2015. Rumania. Disponible en: http://www.ijmd.ro/articole/451 03\%20lrina\%2 OMANOILESCU.pdf

4.-Vargas Alvarado E. Medicina Legal. Cap. 15. 5 ed. México. Trillas 2015. Pág. 195.

5.-DiMaio VJ, DiMaio D. Asphyxia. In: Geberth VJ, series editor. Forensic pathology, 2nd Edn. Boca Ratón, FL: CRC Press, 2001; 229-277.

6.-Concheiro Carro L, Suárez Peñaranda JM. Asfixias mecánicas. en: Villanueva E.(Ed), Medicina Legal y Toxicología. Gisbert Calabuig. $6^{\circ}$ Ed. Masson. Barcelona. 2004. Pág.460-478.

7.-Saukko P, Knight B. Knight's Forensics Pathology. Suffocation and Asphyxia. CRC Press. Cap. 14. Boca Ratón, FL. 2016; Pag.353.

8.-Sauvageau A. Boghossian B.Sc. Classification of Asphyxia: The need for standardrization. Journal of Forensic Sciences Vol.55 Issue. 55 pag. 12591267.Canada.2010. Disponible en: https://onlinelibrary.wiley.com/doi/pdf/10.1111 Li.1556-4029.2010.01459.

9.-Joo Young N. A Classification of Asphyxia Autopsy Cases of the Korea in 2012 according to New Classification of Asphyxia. Korean J Leg Med. 2014 Feb;38(1):8-12.

10.-Idris $\mathrm{AH}$, et al. Recommended guidelines for uniform reporting of data from drowning: the "Utstein style." Circulation.2003;108:2565-2574. doi: 10.1161/01.CIR.0000099581.70012.68.

11.-Idris AH et al, 2015 Revised Utstein-Style Recommended Guidelines for Uniform Reporting of Data From Drowning-Related Resuscitation: An ILCOR Advisory Statement. Doi: 
https://doi.org/10.1161/HCQ.000000000000002 4Circulation: Cardiovascular Quality and Outcomes. 2017;10:e000024. Originally published July 17,2017

12.-Byard R.W. Immersion deaths and drowning: issues arising in the investigation of bodies recovered from water. Forensic Sci Med Pathol (2015) 11: 323. https://doi.org/10.1007/s12024014-9564-5

13.-Schilling UM1, Bortolin M. Drowning. Minerva Anestesiol. 2012 Jan;78(1):69-77. Epub 2011 May 30.

14.-McEwen BJ, Gerdin J.Veterinary Forensic Pathology: Drowning and Bodies Recovered From Water. Vet Pathol. 2016 Sep;53(5):1049-56. doi: 10.1177/0300985815625757. Epub 2016 Feb 29. 15.-Lunetta P, Modell JH. Macroscopical, microscopical, and laboratory findings in drowning victims: a comprehensive review. In: Tsokos M, ed. Forensic Pathology Reviews. Totowa, NJ: Humana Press; 2005:3-77.

16.-Bierens J, Lunetta Ph, Tipton M, Warner D . Physiology of Drowning: A Review. PHYSIOLOGY 31: 147-166, 2016. Published February 17, 2016; doi:10.1152/physiol.00002.2015

17.-Byard RW. Sudden death in the young. 3rd ed. Cambridge: Cambridge University Press; 2010.

18.- Romero Polanco JL. Muertes por sumersión: Revisión y actualización de un tema clásico de la medicina forense. Cuad. med. forense[Internet]. 2007 Jul [citado 2018 Jul 14]; (48-49): 99-130. Disponible

http://scielo.isciii.es/scielo.php?script=sci arttex t\&pid=S1135-76062007000200001\&lng=es.

19.-DelPozo-Luengo M. Marcadores bioquímicos séricos en la muerte por sumersión. Eficacia diagnostica del estroncio y otros elementos traza. Universidad de Murcia, tesis doctoral 2013, [Internet]. 2013 [citado $2018 \mathrm{Jul}$ 20] disponible en:

https://digitum.um.es/xmlui/bitstream/10201/3 5498/1/TESIS\%20DOCTORAL\%20-
\%20M\%C2\%AA\%20SOLEDAD\%20DEL\%2OPOZO\% 20LUENGO.pdf

20.-Síbon Olano A. y col. Síndrome de asfixia por sumersión. Cuad de Medicina Forense; 11(41):229-233.

21.-Tse R. et al. La precisión diagnóstica potencial de la autopsia de los pesos pulmonares, la relación pulmón-corazón y la relación cuerpopulmón en las muertes por ahogamiento. J Forensic Med Pathol. Septiembre de 2018; 39 (3): 223-228. doi: 10.1097 / PAF.0000000000000402. 22.-Blanco Pampín J, García Rivero SA, Tamayo NM, Hinojal Fonseca R. Gastric mucosa lesions in drowning: its usefulness in forensic pathology.Leg Med (Tokyo). 2005 Mar;7(2):89-95.

23.- Zátopková L 1 , Hejna P., Janík M Tinción hemolítica del endocardio de las cámaras del corazón izquierdo: un nuevo signo para el diagnóstico de autopsia de ahogamiento de agua dulce. Forense Sci Med Pathol. 2015 Mar; 11 (1): 65-8. doi: 10.1007 / s12024-014-9620-1

24.-Byard RW. Aortic intimal staining in drowning. Forensic Sci Med Pathol. 2014. doi: 10.1007/s12024-014-9563-6.

25.- Rexon et al. Elevated postmortem vitreous sodium and chloride level in salt water drowning death self-conteined underwater breathing apparatus diving with diving with diving mask in place case report. Am Journal of Forensic Med and Path. 2018. Disponible en: https://journals.Iww.com/amjforensicmedicine/ Abstract/publishahead/Elevated PostmortemVit reous Sodium and Chloride.99240.aspx

26.-Sun CH, Wang B, Li ZD, Qin ZQ. Progress on Diatom Test in Drowning Cases. Fa Yi Xue Za Zhi. 2015, Dec;31(6):462-5[Article in Chinese], abstract, disponible en: https://www.ncbi.nlm.nih.gov/pubmed/271418 $\underline{06}$

27.-Zivkovic $V$. et al. Svechnikov's sign as an indicator of drowning in immersed bodies changed by decomposition: an autopsy study. Forensic sci med pathol. 2013 Springer Science. Disponible en: 
https://link.springer.com/article/10.1007/s1202 4-012-9397-z

28.- Niles NR. Hemorrhage in the middle-ear and mastoid in drowning.Am J Clin Pathol. 1963 Sep;40:281-3.

29.-Robbins RD., Sekhar HK., Siverls V. Temporal bone histopathologic findings in drowning victims. Arco Otolaryngol Head Neck Surg. Septiembre de 1988; 114 (9): 1020-3

30.-Thapar R. et al. Pink tooth phenomenon: An enigma, Journal of Forensics and Legal Medicine 20 (2013) 912-914

31.-Manoilescu I. et al. Post-mortem changes in teeth-forensic issues. International Journal of Medical Dentistry. 2015. Rumania. Disponible en: http://www.ijmd.ro/articole/451 03\%20lrina\%2 OMANOILESCU.pdf

32.-Introna F et-al. Migrant death and the Kater Radez I wreck: from recovery of the relict to marine taphonomic findings and identification of the victims. International Journal of Legal Medicine. Volume 4. 2013. Italia. Disponible: https://link.springer.com/article/10.1007/s0041 4-012-0807-2
33.-De Donno A. et al. Case review, Bodies in sequestered and non-sequestered aquatic enviroments: a comparative taphonomic study using decompositional scoring system. Science and Justice. Italia. 2014. Disponible en: http://www.sciencedirect.com/science/article/pi i/S1355030614001324

34.-Ayaka S., Hisako S., Namiko I., Hirotaro I. The Effects of Racemization Rate for Age Estimation of Pink Teeth. J Forensic Sci, March 2015, Vol. 60, N.2

35.- Solano G. Asfixias mecánicas. Medicina Legal de Costa Rica. Vol. 25. No.2 Scielo. Costa Rica. 2008 Disponible en: http://www.scielo.sa.cr/scielo.php?script=sci ar ttext\&pid=S1409-00152008000200007

36.-Lunetta P, Penttila A, Sajantila A. Circunstancias y hallazgos macropatológicos en 1590 casos consecutivos de cuerpos encontrados en el agua. Am J Forensic Med Pathol. 2002 Dec; 23 (4): 371-6. 